\title{
PI3K/AKT/Afadin signaling pathway contributes to pathological vascularization in glioblastomas
}

\author{
XUAN ZHAI $^{1,2}$, YINGLIANG LI ${ }^{1,2}$, PING LIANG ${ }^{1,2}$, LUSHENG LI $^{1,2}$, YUDONG ZHOU $^{1,2}$, \\ WEIDAN ZHANG ${ }^{1,2}$, DIFEI WANG ${ }^{1,2}$ and GUANGHUI WEI ${ }^{2,3}$
}

\begin{abstract}
${ }^{1}$ Department of Neurosurgery, Children's Hospital of Chongqing Medical University; ${ }^{2}$ Ministry of Education Key Laboratory of Child Development and Disorders, Children's Hospital of Chongqing Medical University; ${ }^{3}$ Department of Urinary Surgery, Children's Hospital of Chongqing Medical University, Chongqing 400014, P.R. China
\end{abstract}

Received September 25, 2016; Accepted October 6, 2017

DOI: 10.3892/ol.2017.7461

\begin{abstract}
Glioblastomas are brain tumors with extensive vascularization that are associated with tumor malignancy. The phosphoinositide 3-kinase (PI3K)/protein kinase B (AKT) signaling pathway is activated in endothelial cell tumors, although its exact function in glioblastoma neovascularization is poorly characterized. The present study identified that endothelial cells derived from human glioblastomas exhibit increased permeability and motility compared with normal brain vascular endothelial cells. Furthermore, the phosphorylation of AKT was significantly induced in glioblastoma-derived endothelial cells and glioblastoma vessels. To the best of our knowledge, the present study demonstrated for the first time that the cell-cell adhesion junction protein Afadin is phosphorylated and re-localized in glioblastoma-derived endothelial cells, and the phosphorylation and re-localization of Afadin is PI3K/AKT pathway-dependent. AKT-mediated phosphorylation and re-localization of Afadin may be critically involved in the modulation of brain endothelial permeability and migration. Therapies targeting the PI3K/AKT/Afadin pathway may therefore be beneficial for reducing the angiogenic potential of glioblastoma.
\end{abstract}

\section{Introduction}

Glioblastomas (GBMs) are brain tumors with extensive vascularization that are associated with tumor malignancy (1). Tumor vasculature is structurally and functionally abnormal, and the

Correspondence to: Dr Xuan Zhai, Department of Neurosurgery, Children's Hospital of Chongqing Medical University, 136 Zhongshan 2nd Road, Yuzhong, Chongqing 400014, P.R. China

E-mail: zhaixuan@163.com

Dr Guanghui Wei, Department of Urinary Surgery, Children's Hospital of Chongqing Medical University, 136 Zhongshan 2nd Road, Yuzhong, Chongqing 400014, P.R. China

E-mail: ghwei1963@126.com

Key words: glioblastoma, protein kinase B signaling, Afadin, vascularization, endothelial cells vessels are highly permeable, tortuous, dilated, saccular and are poorly covered by pericytes (2). Excessive blood leakage associated with vessel immaturity increases the interstitial pressure in the tumor and facilitates invasive tumor cell penetration into the circulation, allowing the metastatic spread of cancer (3). A key feature of GBMs is the extensive network of abnormal vasculature, characterized by glomeruloid structures and endothelial hyperplasia (4). Due to the location of GBM in the brain, the primary clinical problem is the edematous swelling and dramatic increase of intracerebral pressure caused by the disrupted blood brain barrier (BBB) and leaky blood vessels $(5,6)$. A current therapeutic approach against GBMs is targeted against this vascularization process (7). Targeting endothelial cells (ECs) is a major focus of anti-vascular therapy (1). However, targeting glioma angiogenesis by vascular endothelial growth factor inhibition has only exhibited a minor effect on patient survival (8). At present, the biological characteristics of ECs in GBMs are poorly characterized, and the cellular and molecular mechanisms underlying the vascularization in GBMs require additional study.

Endothelial cell-cell junctions are critical for vascular integrity, EC growth, vascular development and the maintenance of vascular function (9). Endothelial junctional proteins also control multiple signaling events intracellularly via membrane proteins, clustering of signaling molecules and growth factor receptors (10). Afadin, originally identified as an actin-binding protein, is localized at adherens junctions and is expressed in epithelial cells, neurons, fibroblasts and ECs $(11,12)$. Afadin is an adaptor protein that binds to numerous scaffolding and actin-binding proteins, and contributes to the association of immunoglobulin-like cell adhesion molecule nectins with other cell-cell adhesion molecules and intracellular signaling systems (11). Afadin has multiple functions in cell-cell adhesion and in cell movement, proliferation, survival and polarization (13). Afadin has also been demonstrated to regulate integrin-mediated cell adhesion and cell migration, although it appears that the function of Afadin in the positive or negative regulation of cell motility is context-dependent $(14,15)$. Afadin also directly interacts with the tight junction-associated protein junction adhesion molecule-A, thereby affecting tight junction assembly during progression of the polarity program (16). 
The phosphoinositide 3-kinase (PI3K)/protein kinase B (AKT) pathway is a complex and central regulator of a number of fundamental cellular processes (17) and regulates all phenotypes that contribute to the progression of human cancer, including GBM $(17,18)$. The AKT pathway was previously demonstrated to be activated in EC tumors $(19,20)$. Sustained endothelial AKT activation mediated increased blood vessel size and generalized edema caused by chronic vascular permeability (21). AKT signaling in the tumor vasculature was sensitive to rapamycin, suggesting that rapamycin may affect tumor growth in part by acting as a vascular AKT inhibitor (21). A previous study indicated that Afadin is phosphorylated by all AKT isoforms and that this phosphorylation elicits a re-localization of Afadin from the adherens junctions to the nucleus (15). The present study attempted to clarify the functions of the PI3K/AKT pathway and Afadin in the GBM tumor vasculature.

\section{Materials and methods}

Reagents. The PI3K inhibitor, LY294002 (Calbiochem; Merck KGaA, Darmstadt, Germany) was used at a final concentration of $10 \mu \mathrm{M}$. The antibodies used for immunostaining and western blotting were anti-cluster of differentiation (CD) 31 (cat. no. 910003; BioLegend, Inc., San Diego, CA, USA), anti-Afadin (cat. no. ab11337; Abcam, Cambridge, UK), anti-phosphorylated (phospho)-Afadin Ser1718 (cat. no. 5485; Cell Signaling Technology, Inc., Danvers, MA, USA), anti-AKT (cat. no. 9272; Cell Signaling Technology, Inc.), anti-phospho-AKT Ser473 (cat. no. 4060; Cell Signaling Technology, Inc.), anti-vascular endothelial (VE)-cadherin (cat. no. AF938; R\&D Systems, Inc., Minneapolis, MN, USA), anti-p-endothelial nitric oxide synthase (cat. no. 9571; Cell Signaling Technology, Inc.), anti-p-ERK (cat. no. 9101; Cell Signaling Technology, Inc.) and anti-ERK (cat. no. 4695; Cell Signaling Technology, Inc.).

Isolation of primary ECs. GBM or general traumatic surgical specimens were collected in accordance with a protocol approved by the Ethics Committee of Clinical Investigation of Chongqing Medical University (Chongqing, China). These specimens were obtained subsequent to the procurement of written informed consent by the neurosurgical team at the Children's Hospital of Chongqing Medical University (Chongqing, China). Surgical samples from 9 GBM patients ( 7 males and 2 females) were included. The median age was 9 years and the age range was between 2 and 13 years. Inclusion criteria included confirmation of a histopathological diagnosis of classic GBM by two independent pathologists (Chongqing Medical University). Pediatric patients with histopathological diagnosis of other types of tumor were excluded from the present study. Control human brain vascular ECs (HBVECs) were obtained from 5 pediatric brain traumatic cases that had not previously been diagnosed with cancer. All tissues were collected between January and November 2014. ECs were derived from GBM tissues (GBM-ECs) or general traumatic surgical specimens (HBVECs) and characterized as previously described (22). Briefly, GBM tumors or general traumatic brain tissues were disaggregated using $1-\mathrm{mg} / \mathrm{ml}$ collagenase IV (Sigma-Aldrich; Merck KGaA) for $60 \mathrm{~min}$ at $37^{\circ} \mathrm{C}$. Isolated cells and vessel fragments were passed through a $70-\mu \mathrm{m}$ mesh, collected by centrifugation at
$800 \mathrm{x} \mathrm{g}$ for $10 \mathrm{~min}$ at $4^{\circ} \mathrm{C}$, and seeded into 100 -mm dishes with modified M-199 medium (9.87 g/l medium M-199; cat. no. 11150059; Thermo Fisher Scientific, Inc., Waltham, MA, USA), $10 \mathrm{ml} / 1$ 100X basal medium Eagle vitamin solution (cat. no. 21010046; Thermo Fisher Scientific, Inc.), 1 g/l glucose and $2.2 \mathrm{~g} / 1 \mathrm{NaHCO}_{3}$ containing $20 \%$ heat-inactivated low-endotoxin fetal bovine serum (Thermo Fisher Scientific, Inc.), $100 \mu \mathrm{g} / \mathrm{ml}$ neomycin, $20 \mathrm{U} / \mathrm{ml}$ nystatin and $2 \mathrm{mmol} / \mathrm{l}$ L-glutamine (complete medium), and cultured for a week at $37^{\circ} \mathrm{C}$ with $5 \% \mathrm{CO}_{2}$. Culture medium was changed twice a week. To purify the ECs, the cultured cells were subjected to fluorescence-activated cell sorting for CD31-positive cells using fluorescein isothiocyanate (FITC)-conjugated anti-CD31 (1:100; cat. no. 303103, BioLegend, Inc.). Cells were incubated with anti-CD31 antibody at $400 \mu \mathrm{g} / \mathrm{ml}$ for $30 \mathrm{~min}$ at $4^{\circ} \mathrm{C}$ at a volume of $2 \mu \mathrm{l}$ of antibody per $10^{5}$ cells. The sorted cells were then collected in EC growth medium (EGM2; Lonza Group Ltd., Basel, Switzerland), plated in 24-well tissue culture plates and incubated at $37^{\circ} \mathrm{C}$ with $5 \% \mathrm{CO}_{2}$.

Immunocytofluorescence analysis. Cells were grown on Lab-Tek II chamber slide systems (Thermo Fisher Scientific, Inc.), fixed with $4 \%$ paraformaldehyde in phosphate buffered saline (PBS; pH 7.4) overnight at $4^{\circ} \mathrm{C}$, rinsed with PBS and blocked with PBS containing 5\% BSA (cat. no. A2058; Sigma-Aldrich; Merck KGaA) for $30 \mathrm{~min}$ at room temperature. Primary antibodies against VE-cadherin [cat. no. sc-9989; 1:100 dilution in PBS containing 0.1\% (v/v) Tween-20 (T-PBS); Santa Cruz Biotechnology, Inc., Dallas, TX, USA]; cluster of differentiation (CD) 31 (cat. no. 550389; 1:200; BD Biosciences, Franklin Lakes, NJ, USA); Afadin (cat. no. ab11337; 1:100; Abcam) and phospho-AKT Ser473 (cat. no. 4060; 1:300; Cell Signaling Technology, Inc.) were applied to the surface of the slide and incubated overnight at $4^{\circ} \mathrm{C}$. The following day, cells were incubated with secondary antibodies Alexa Fluor 594- or 488-conjugated goat anti-rabbitimmunoglobulin $\mathrm{G}(\mathrm{IgG})$ [heavy and light $(\mathrm{H}+\mathrm{L})]$ or anti-mouse $\operatorname{IgG}(\mathrm{H}+\mathrm{L})$ (cat. no. A-11037 and A-11001; 1:500; Thermo Fisher Scientific, Inc.) for $1 \mathrm{~h}$ at room temperature. Finally, cells were counterstained with DAPI (1:5,000 dilution in T-PBS; Sigma-Aldrich; Merck KGaA) for $10 \mathrm{~min}$ at room temperature. Negative controls, consisting of secondary antibodies alone, were performed on each section of tissue studied. Immunofluorescence photomicrographs were captured using a fluorescence microscope (Nikon Corporation, Tokyo, Japan).

EC low-density lipoprotein ( $L D L)$ uptake. The ability of isolated ECs to take up acetylated (Ac) -LDL was examined using Ac-LDL labeled with 1,1'-dioctadecyl-3,3,3',3'-tetramethylindocarbocyanine perchlorate (Dil). The primary cells were seeded at an initial density of $2 \times 10^{5}$ cells $/ \mathrm{cm}^{2}$. Cultures grown on Lab-Tek II chamber slide systems (cat. no. 154526 , Thermo Fisher Scientific, Inc.) were incubated with $15 \mu \mathrm{g} / \mathrm{ml}$ Dil-Ac LDL (Thermo Fisher Scientific, Inc.) in EGM2 for 20 min, washed with PBS (pH 7.4) and immediately observed by inverted fluorescence microscopy using a rhodamine filter.

Permeability assay. HBVECs or GBM-ECs ( $2 \times 10^{5}$ cells) were seeded on Transwell filters (0.4- $\mu \mathrm{m}$ pore size, Costar; Corning 
Incorporated, Corning, NY, USA) in 24-well dishes and grown in EGM2 until they reached $100 \%$ confluence. For the inhibition assay, GBM-ECs were pre-incubated for $24 \mathrm{~h}$ in the presence of LY294002 $(10 \mu \mathrm{M})$ or with $1 \mu \mathrm{l}$ of dimethylsulfoxide as a vehicle control. Then, FITC-dextran (70,000 Da; Sigma-Aldrich; Merck $\mathrm{KGaA}$ ) at a final concentration of $1 \mathrm{mg} / \mathrm{ml}$ was added to the upper chamber. At $30 \mathrm{~min}, 50 \mu 1$ samples were removed from the lower chamber. The fluorescence of these samples was measured at an excitation wavelength of $485 \mathrm{~nm}$ and an emission wavelength of $530 \mathrm{~nm}$ using a fluorescence plate reader (BioTek Instruments, Inc., Winooski, VT, USA).

Transwell migration assay. Cell migration was evaluated using a Transwell system (Costar; Corning Incorporated) comprised of $8-\mu \mathrm{m}$ polycarbonate filter inserts in 24-well plates. Briefly, HBVECs and GBM-ECs (passaged 2-3 times) were harvested using trypsin in EGM2. Next, $600 \mathrm{ml}$ EGM2 medium was added to the lower chambers, while $1 \times 10^{5}$ HBVECs or GBM-ECs were plated in the upper chambers in EGM2. After $4 \mathrm{~h}$ of incubation, cells on the bottom of the Transwell membrane were fixed with $4 \%$ paraformaldehyde at $37^{\circ} \mathrm{C}$ for $20 \mathrm{~min}$ and stained with $1 \%$ crystal violet at $37^{\circ} \mathrm{C}$ for $10 \mathrm{~min}$; the non-migrating cells in the upper chamber were removed with blunt-end swabs. The membranes were washed three times with PBS and images were captured under a fluorescence microscope (Olympus Corporation, Tokyo, Japan). Finally, the level of cell migration was quantified by counting four fields of view. The mean count of triplicated chambers is presented.

Western blot analysis. HBVECs and GBM-ECs were cultured in 6-cm dishes. Proteins were extracted using 2X SDS sample buffer (Sigma-Aldrich; Merck KGaA) and quantified using a Bradford protein assay kit (P0006C; Beyotime Institute of Biotechnology). The extracted proteins (30 $\mu \mathrm{g} / \mathrm{lane})$ were separated using 4-15\% Protean ${ }^{\circledR}$ TGX $^{\mathrm{TM}}$ Precast Protein gels (cat. no. 4561083; Bio-Rad Laboratories, Inc., Hercules, CA, USA). Proteins were transferred onto polyvinylidene difluoride membranes. Following blocking with $5 \%$ bovine serum albumin overnight at $4^{\circ} \mathrm{C}$, the membranes were incubated with the aforementioned antibodies overnight at $4^{\circ} \mathrm{C}$. anti-Afadin $(1: 1,000$; cat. no. ab11337; Abcam, Cambridge, UK), anti-phosphorylated (phospho)-Afadin Ser1718 (1:1,000; cat. no. 5485; Cell Signaling Technology, Inc., Danvers, MA, USA), anti-AKT (1:1,000; cat. no. 9272; Cell Signaling Technology, Inc.), anti-phospho-AKT Ser473 (1:1,000; cat. no. 4060; Cell Signaling Technology, Inc.), anti-p-endothelial nitric oxide synthase (1:1,000; cat. no. 9571 ; Cell Signaling Technology, Inc.) and anti-p-ERK (1:1,000; cat. no. 9101; Cell Signaling Technology, Inc.). GAPDH (1:2,500; cat. no. 2118; Cell Signaling Technology, Inc.) was used as loading control. Membranes were subsequently incubated with horseradish peroxidase-conjugated anti-mouse and anti-rabbit secondary antibodies (cat. nos. ab97040 and ab7090; 1:2,500; Abcam) for $1 \mathrm{~h}$ at room temperature. Following two membrane washes, membranes were developed with 3,3'-diaminobenzidine tetrahydrochloride and imaged/quantified using a Sigma-Aldrich microDOC gel documentation system (cat. no. Z692557; Merck KGaA).

Statistical analysis. Independent Student's t-tests were used to determine statistical significance. Statistical tests were
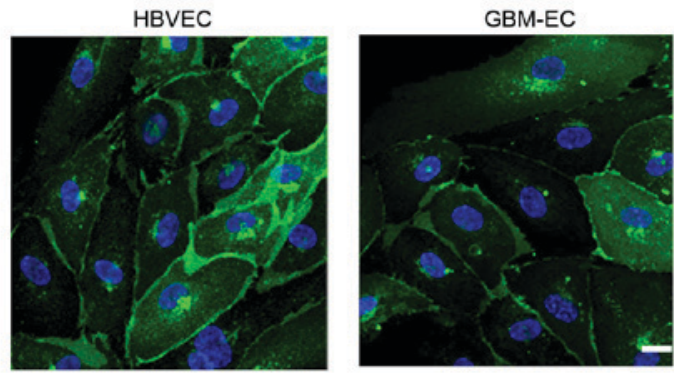

B
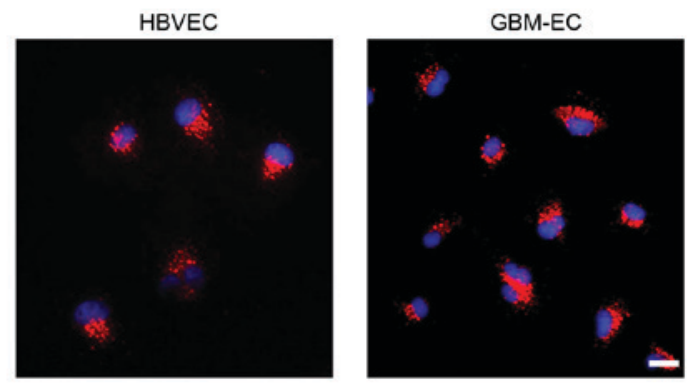

$\mathrm{C}$

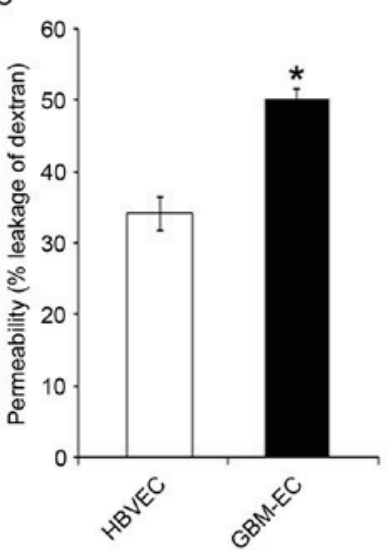

D

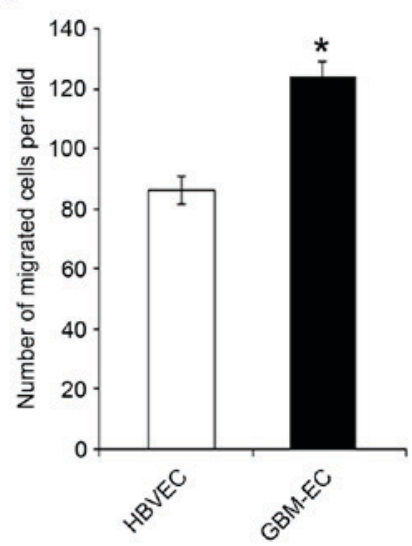

Figure 1. Increased permeability and motility in GBM-EC. (A) Cluster of differentiation 31 (green) immunostaining of primary isolated brain ECs from normal HBVECs and GBM-ECs. Nuclei were stained with DAPI (blue) (B) Accumulation of acetylated low-density lipoprotein (red) by primary HBVECs and GBM-ECs. (C) HBVECs and GBM-ECs were grown to confluence on Transwell filters. Permeability for FITC-dextran (70 kDa) was measured using a fluorescence plate reader ( $\lambda$ excitation $485 \mathrm{~nm} ; \lambda$ emission $530 \mathrm{~nm}$ ). (D) A Transwell motility assay using HBVCEs and GBM-ECs was performed using 8- $\mu \mathrm{m}$ Transwell chambers. Migrated cells were quantified by counting crystal violet stained cells in 4 random fields of view/filter. Results are the mean \pm standard error of three independent experiments. Scale bars, $10 \mu \mathrm{m} .{ }^{*} \mathrm{P}<0.05$ vs. HBVECs. HBVEC, human brain vascular endothelial cells; GBM-ECs, glioblastoma endothelial cells; FITC, fluorescein isothiocyanate.

performed in Microsoft Excel 2013 (Microsoft Corporation, Redmond, WA, USA). All P-values were derived from at least three independent experiments. $\mathrm{P}<0.05$ was considered to indicate a statistically significant difference.

\section{Results}

ECs isolated from human GBM tumors demonstrate increased permeability and motility. To characterize the phenotype of GBM-derived ECs, primary GBM-ECs and HBVECs were isolated from 9 and 5 patients, respectively. CD31 immunostaining (Fig. 1A) and uptake of Dil-acetylated LDL (Fig. 1B) demonstrated the purity of primary isolated brain ECs. The 
A
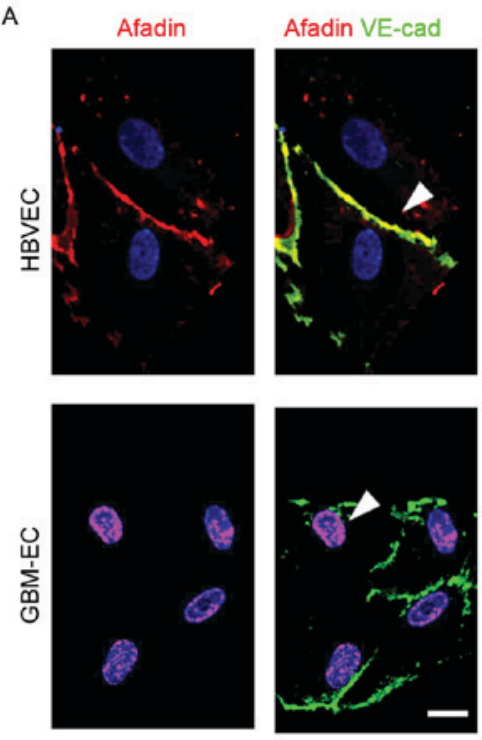

C

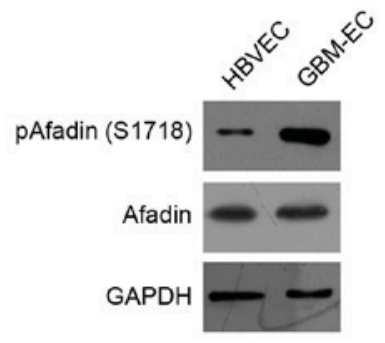

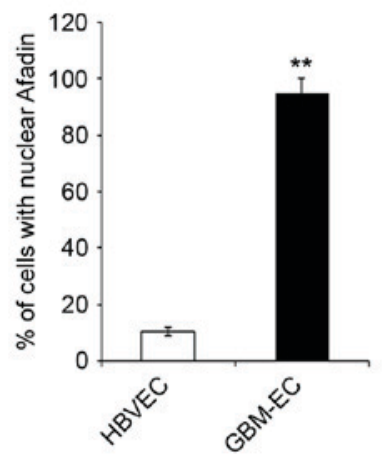
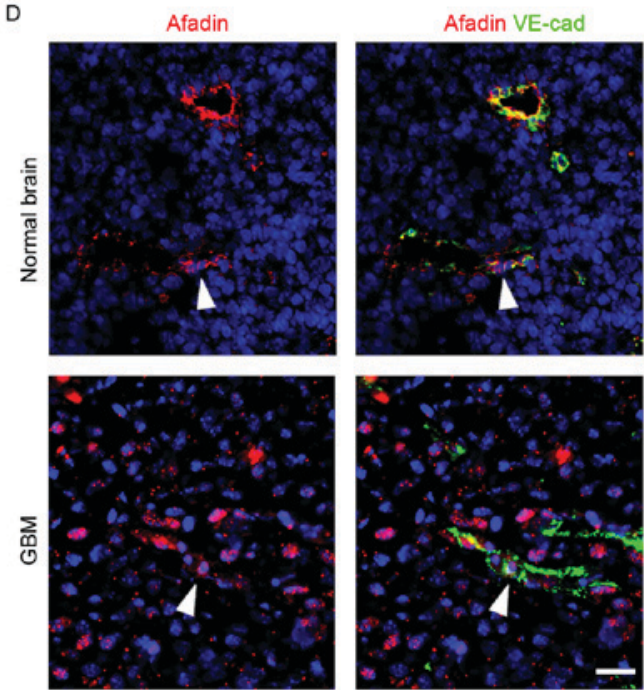

Figure 2.Immunocytofluorescence analysis and western blot assay detecting phosphorylation and localization of Afadin. (A) Immunofluorescence was performed with the indicated antibodies: Afadin (red); VE-cadherin (green); and DAPI (blue). HBVECs exhibited Afadin cell junction distribution (arrow), while GBM-ECs exhibited Afadin nuclear location (arrow). Images are representative of multiple fields of view and of three independent experiments. Scale bars, $10 \mu \mathrm{m}$. (B) Quantification of the nuclear staining is presented in the bar graph. Error bars are the mean \pm standard error. (C) Western blotting indicated the phosphorylation and expression of Afadin in HBVECs and GBM-ECs. (D) Afadin demonstrated nuclear localization in GBM vessels (arrows) as compared with cell membrane distribution in normal brain tissue (arrows). Afadin (red), VE-cadherin (green) and DAPI (blue). Representative images are presented, with scale bars, $50 \mu \mathrm{m} .{ }^{* *} \mathrm{P}<0.01 \mathrm{vs}$. HBVECs, assessed using Student's t-test. VE-cad, vascular endothelial cadherin; HBVEC, human brain vascular endothelial cells; GBM-ECs, glioblastoma endothelial cells.

results indicate that these two types of ECs were positive for CD31, and that GBM-ECs exhibit a similar morphology and LDL uptake activity to HBVECs (Fig. 1A and B). Permeability of the EC barrier was assessed by comparing the transport of $70 \mathrm{kDa}$ fluorescent dextran across the endothelial monolayer. HBVECs or GBM-ECs were seeded into the upper chamber of $0.4 \mu \mathrm{m}$ Transwell filters. The degree of leakage was determined by measuring the intensity of FITC-dextran fluorescence in the lower chamber. The results demonstrate that FITC-dextran leakage was significantly increased in GBM-ECs compared with HBVECs (Fig. 1C), indicating significantly disrupted endothelial barrier function in GBMs. Cell motility is of particular interest in the design of anti-cancer therapeutics, as endothelial migration is required for tumor angiogenesis (23). Next, EC migration was evaluated using the Transwell migration assay. HBVECs or GBM-ECs were plated onto the upper chambers of an $8-\mu \mathrm{m}$ Transwell system. Cells were allowed to migrate for $4 \mathrm{~h}$ through the $8-\mu \mathrm{m}$ pores of the polycarbonate filters. As indicated in Fig. 1D, compared with HBVECs, a significantly increased number of GBM-ECs migrated to the lower side of the filter. This result demonstrated that endothelial migration is induced in GBMs.
Adherens junction protein Afadin is phosphorylated and re-localized in GBM-ECs. The integrity of intercellular junctions is a major determinant of permeability of the brain endothelium (24). The adherens junction protein, Afadin, participates in the initial step of junction formation and serves a fundamental role in the regulation of cell-cell contact (25). The expression and localization of Afadin was detected in ECs Immunofluorescence microscopy indicated that total Afadin exhibited a predominantly membrane-restricted localization in HBVECs and was concentrated at the cell-cell contact sites between adjacent HBVECs, which was co-localized with a marker of endothelial cell-cell contact, VE-cadherin (Fig. 2A). However, Afadin membrane localization and its distribution along the cell borders was markedly decreased, accompanied by the appearance of its nuclear localization in GBM-ECs (Fig. 2A). Quantification of the nuclear translocation was evaluated using co-staining with DAPI and this revealed that a significantly higher percentage of GBM-ECs stained positive for nuclear Afadin compared with HBVECs (Fig. 2B). Notably, western blot analysis revealed that total Afadin protein expression did not change in GBM-ECs, while phosphorylation of Afadin at serine 1718 (Ser1718) was markedly induced in 

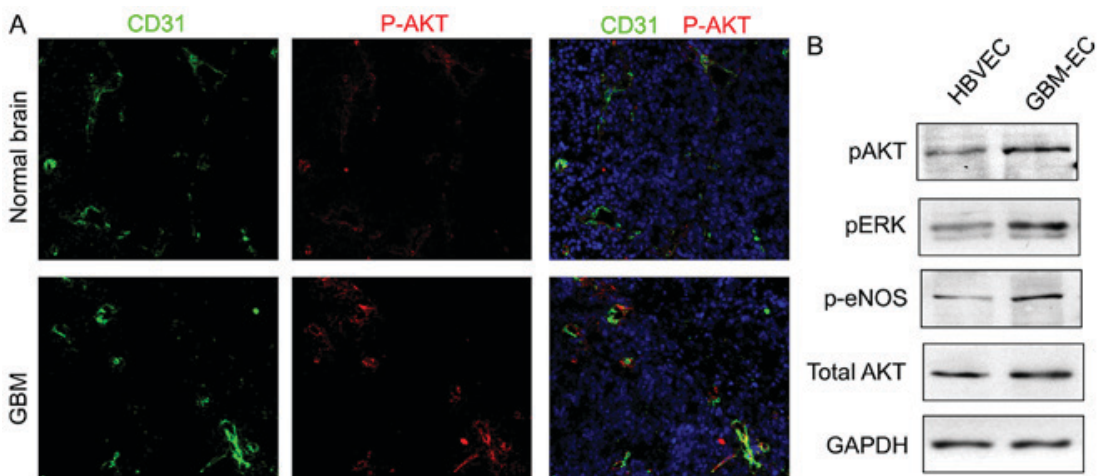

Figure 3. Immunocytofluorescence analysis and western blot assay detecting phosphorylation of AKT. (A) Phosphorylation levels of AKT in normal brain vessels and GBM vessels. P-AKT (red), CD31 (green) and DAPI (blue). Representative images are presented. Scale bars, $100 \mu \mathrm{m}$. (B) Western blotting demonstrated phosphorylation levels of AKT, ERK, eNOS, and total expression level of AKT in HBVECs and GBM-ECs. p, phosphorylated; AKT, protein kinase B; CD31, cluster of differentiation; ERK, extracellular signal-related kinase; eNOS, endothelial nitric oxide synthase; HBVEC, human brain vascular endothelial cells; GBM-ECs, glioblastoma endothelial cells.
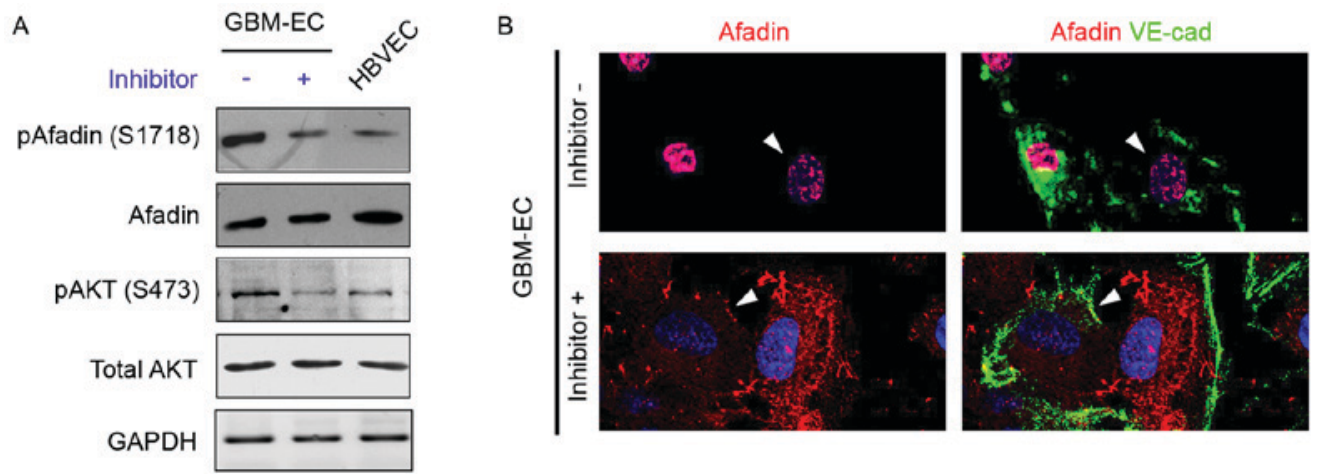

Figure 4. Effects of PI3K/Akt inhibition on phosphorylation and localization of Afadin. (A) Western blotting revealed the phosphorylation and expression of Afadin and AKT in LY294002 inhibitor or dimethylsulfoxide (control)-treated GBM-ECs and HBVECs. (B) Afadin (red) and VE-cad (green) double immunostaining on LY294002 inhibitor-treated GBM-ECs exhibited Afadin cell membrane and junction distribution (arrows), while control GBM-ECs exhibited Afadin nuclear location (arrows). VE-cad, vascular endothelial cadherin; HBVEC, human brain vascular endothelial cells; GBM-ECs, glioblastoma endothelial cells; p, phosphorylated; AKT, protein kinase B.

GBM-ECs compared with HBVECs (Fig. 2C). Finally, Afadin localization in human GBMs was assessed. GBM tumors and normal brain tissue obtained from archival pathology specimens were used for localization analysis and evaluated using immunofluorescence. Afadin expression in ECs was assessed by co-immunostaining for Afadin and VE-cadherin. A marked increase in the nuclear localization of Afadin in the ECs of GBM vessels compared with normal brain tissue was identified. Fig. 2D presents representative images of normal brain tissue and GBM specimens. Taken together, these results suggest that Afadin is phosphorylated and re-localized in GBM-ECs.

AKT pathway is activated in GBM-ECs. A previous study has indicated that the PI3K/AKT pathway serves a critical function in the pathogenesis of vasculature and BBB interruption in tumors (26). Whether the PI3K/AKT pathway is activated in human GBM-ECs was next examined. To investigate the level of AKT activation, AKT phosphorylation was analyzed using the immunofluorescence staining of human GBMs. The results demonstrated that numerous tumor ECs expressed phospho-AKT in GBMs, while quiescent vessels in the normal brain did not exhibit a high intensity of phospho-AKT
(Fig. 3A). Western blot analyses of cell lysates confirmed the immunostaining results by demonstrating elevated levels of phospho-AKT, standardized to the level of total AKT, in GBM-ECs compared with HBVECs (Fig. 3B). Additionally, AKT activation was associated with the activation of downstream signaling pathways, as evidenced by the increased phosphorylation of extracellular signal-related kinase and endothelial nitric oxide synthase (Fig. 3B). These results demonstrated that the PI3K/AKT pathway is activated in GBM-ECs.

Phosphorylation and re-localization of Afadin is regulated by the PI3K/AKT pathway in GBM-ECs. A previous study demonstrated that PI3K/AKT signaling phosphorylates Afadin at Ser1718 in cancer epithelial cells (15); therefore, our group hypothesized that the induction of Afadin phosphorylation and re-localization is mediated by the PI3K/AKT pathway in GBM-ECs. To examine this hypothesis, GBM-ECs were treated with the PI3K inhibitor LY294002 $(10 \mu \mathrm{M})$ for $4 \mathrm{~h}$, which decreased AKT activity (Fig. 4A). The inhibition of AKT in GBM-ECs prevented the Ser1718-phosphorylation of Afadin (Fig. 4A). The effect of AKT on Afadin localization was investigated. Notably, the nuclear localization of Afadin 
A

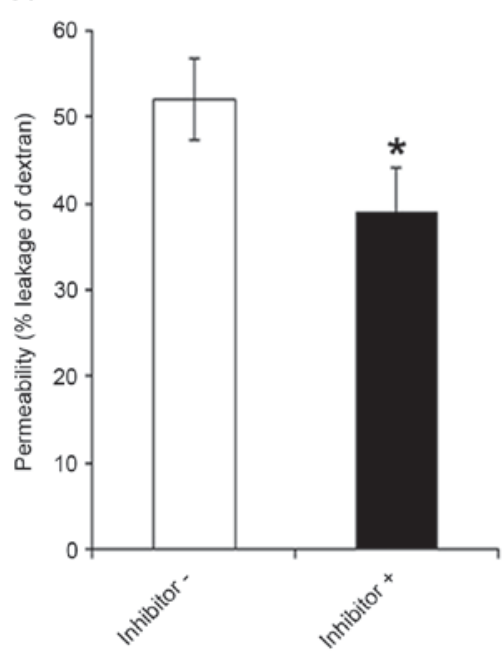

B

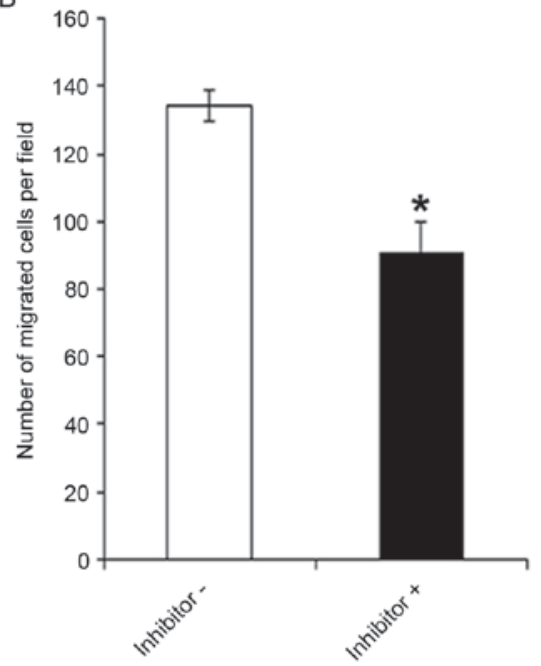

GBM-EC

Figure 5. Permeability assay and Transwell migration assay. (A) GBM-ECs were pretreated with LY294002 inhibitor or DMSO as control. Then, the cells were grown to confluence on Transwell filters. Permeability for fluorescein isothiocyanate-dextran $(70 \mathrm{kDa})$ was measured using a fluorescence plate reader $(\lambda$ excitation $485 \mathrm{~nm} ; \lambda$ emission $530 \mathrm{~nm}$ ). (B) Inhibitor or DMSO (control)-treated GBM-ECs were plated onto the upper chamber of an 8- $\mu \mathrm{m}$ Transwell system. Cells were allowed to migrate for $4 \mathrm{~h}$. Migrated cells were quantified by counting crystal violet-stained cells in 4 random fields/filter. Results are presented as the mean \pm standard error of 3 independent experiments. ${ }^{*} \mathrm{P}<0.05$ vs. negative control. GBM-ECs, glioblastoma endothelial cells; DMSO, dimethylsulfoxide.

was reversed by LY29400 treatment in GBM-ECs. Afadin protein accumulated at cell-cell junctions in LY29400-treated GBM-ECs. In contrast, Afadin accumulation was not observed at cell-cell junctions in untreated cells (Fig. 4B). The cellular localization of Afadin in the LY29400-treated GBM-ECs was in agreement with the localization observed in HBVECs.

Effects of the PI3-K/AKT/Afadin pathway on the permeability and migration of GBM-ECs. Whether the PI3K/AKT/Afadin pathway regulates brain endothelial permeability and motility, which are dependent on intact adherens junctions, was next investigated. GBM-ECs were pretreated with LY294002 inhibitor or control vehicle for $4 \mathrm{~h}$. LY294002 exposure significantly decreased the FITC-Dextran leakage of GBM-ECs compared with those treated with the DMSO control (Fig. 5A). As indicated in Fig. 5B, pre-treatment of cells with LY294002 inhibitors significantly reduced GBM-EC migration in the Transwell experiments. Taken together, these results suggest that the nuclear localization of Afadin and phosphorylation at Ser1718 by the AKT pathway is relevant for high permeability and pathological angiogenesis in GBM vessels.

\section{Discussion}

To develop novel and effective strategies against GBMs, which are highly vascularized, it is mandatory to understand the biological characteristics of ECs in GBMs. Considering the different vascular biology of different tumors and organs, and that ECs tend to change their phenotype in response to culture conditions (27), there are significant benefits associated with the use of freshly isolated ECs from GBMs for the investigation of GBM-induced angiogenesis. In the present study, GBM-ECs were established using 9 patient-derived GBM EC cultures and HBVECs were isolated from normal brain tissues. There were no apparent differences in EC morphology between the HBVECs and GBM-ECs. However, a significantly higher permeability and a marked increase in migration of GBM-ECs compared with HBVECs were observed, indicating that ECs are abnormally activated in GBMs.

The intercellular junctions of ECs serve an important barrier function that regulates permeability to small molecules (25). Previous studies have indicated that Afadin regulates migration and cell-cell adhesion in other cell types, including fibroblasts and epithelial cells $(28,29)$. A previous study indicated that Afadin is associated with angiogenesis through Rap1 signaling (30). The present study has demonstrated that Afadin is phosphorylated and re-localized in GBM-ECs compared with HBVECs in human brain tissues and in vitro cultures, suggesting the potential of phosphorylation and nuclear localization of Afadin as a novel marker of pathological angiogenesis in GBMs.

The PI3K/AKT pathway is a frequently unregulated pathway in cancer; with $86 \%$ of GBM clinical samples exhibiting alterations in the receptor tyrosine kinases/PI3K pathway $(31,32)$. Furthermore, sustained endothelial activation of AKT1 has been demonstrated to induce the formation of structurally abnormal blood vessels that recapitulate the aberrations of tumor vessels (31). However, downstream mediators of AKT in brain tumor vasculatures remain poorly understood. AKT induces phosphorylation of Afadin in epithelial cells (15). However, the involvement of AKT-Afadin in angiogenesis is unknown. The present study revealed that the phosphorylation of AKT was significantly induced in GBM-ECs compared with HBVECs, indicating that the PI3K/AKT pathway is activated in GBM-ECs. Notably, to the best of our knowledge, these data demonstrate for the first time that the phosphorylation and re-localization of Afadin is PI3K/AKT pathway-dependent in human brain ECs. The inhibition of AKT by a PI3K inhibitor was revealed to prevent Ser1718-phosphorylation and the nuclear localization of Afadin in GBM-ECs.

The present study demonstrated that AKT-mediated phosphorylation and re-localization of Afadin was critically 
involved in the modulation of brain endothelial permeability. The pharmacological inhibition of AKT activity was associated with a decreased permeability in GBM-ECs, as evidenced by the decreased passage of $70 \mathrm{kDa}$ dextran. GBM-EC migration was revealed to be downregulated by inhibition of AKT activity. Therefore, the present study has identified a function for the PI3K/AKT/Afadin pathway in the regulation of EC permeability and migration. These data on the molecular interactions of the PI3K/AKT pathway and Afadin may assist to explain an important regulatory mechanism of AKT-mediated function, including the control of EC permeability and migration.

In conclusion, the data of the present study demonstrated that AKT-mediated phosphorylation and re-localization of Afadin is an important mechanism for the regulation of GBM endothelial permeability and motility. Dysregulation of AKT activity contributes to Afadin-associated defects in vascular permeability or angiogenesis associated with GBM metastasis or chronic edema. Therefore, treatment with drugs targeting the PI3K/AKT/Afadin pathway may be beneficial in reducing the angiogenic potential of GBMs. Additional studies are required to understand how this pathway is integrated into the various signaling networks that regulate brain EC permeability and migration.

\section{Acknowledgements}

The present study was supported by the special fund of Chongqing Key Laboratory (grant no. cstc2012gg-yyjs0838), from Chongqing Science and Technology Commission Scientific and Technological Projects, and from the Chongqing Natural Science Foundation (grant no. cstc2015jcyjBX0144).

\section{References}

1. Norden AD, Drappatz J and Wen PY: Antiangiogenic therapies for high-grade glioma. Nat Rev Neurol 5: 610-620, 2009.

2. Jain RK: Normalization of tumor vasculature: An emerging concept in antiangiogenic therapy. Science 307: 58-62, 2005.

3. Goel S, Duda DG, Xu L, Munn LL, Boucher Y, Fukumura D and Jain RK: Normalization of the vasculature for treatment of cancer and other diseases. Physiol Rev 91: 1071-1121, 2011.

4. Wang R, Chadalavada K, Wilshire J, Kowalik U, Hovinga KE, Geber A, Fligelman B, Leversha M, Brennan C and Tabar V: Glioblastoma stem-like cells give rise to tumor endothelium. Nature 468: 829-833, 2010.

5. Wolburg H, Noell S, Fallier-Becker P, Mack AF and Wolburg-Buchholz K: The disturbed blood-brain barrier in human glioblastoma. Mol Aspects Med 33: 579-589, 2012.

6. Mrugala MM: Advances and challenges in the treatment of glioblastoma: A clinician's perspective. Discov Med 15: 221-230, 2013.

7. Gerstner ER and Batchelor TT: Antiangiogenic therapy for glioblastoma. Cancer J 18: 45-50, 2012.

8. Pàez-Ribes M, Allen E, Hudock J, Takeda T, Okuyama H, Viñals F, Inoue M, Bergers G, Hanahan D and Casanovas O: Antiangiogenic therapy elicits malignant progression of tumors to increased local invasion and distant metastasis. Cancer cell 15 : 220-231, 2009

9. Bazzoni G and Dejana E: Endothelial cell-to-cell junctions: Molecular organization and role in vascular homeostasis. Physiol Rev 84: 869-901, 2004.

10. Mehta D and Malik AB: Signaling mechanisms regulating endothelial permeability. Physiol Rev 86: 279-367, 2006.

11. Takai Y, Ikeda W, Ogita $\mathrm{H}$ and Rikitake Y: The immunoglobulinlike cell adhesion molecule nectin and its associated protein afadin. Annu Rev Cell Dev Biol 24: 309-342, 2008.
12. Mandai K, Nakanishi H, Satoh A, Obaishi H, Wada M, Nishioka H, Itoh M, Mizoguchi A, Aoki T, Fujimoto T, et al: Afadin: A novel actin filament-binding protein with one PDZ domain localized at cadherin-based cell-to-cell adherens junction. J Cell Biol 139: 517-528, 1997.

13. Ogita H, Rikitake Y, Miyoshi J and Takai Y: Cell adhesion molecules nectins and associating proteins: Implications for physiology and pathology. Proc Jpn Acad Ser B Phys Biol Sci 86: 621-629, 2010.

14. Su Li,Hattori M,Moriyama M,Murata N,Harazaki M,Kaibuchi K and Minato N: AF-6 controls integrin-mediated cell adhesion by regulating Rap1 activation through the specific recruitment of Rap1GTP and SPA-1. J Biol Chem 278: 15232-15238, 2003.

15. Elloul S, Kedrin D, Knoblauch NW, Beck AH, Toker A: The adherens junction protein afadin is an AKT substrate that regulates breast cancer cell migration. Mol Cancer Res 12: 464-476, 2014.

16. Takai $\mathrm{Y}$ and Nakanishi H: Nectin and afadin: Novel organizers of intercellular junctions. J Cell Sci 116: 17-27, 2003.

17. Arcaro A and Guerreiro AS: The phosphoinositide 3-kinase pathway in human cancer: Genetic alterations and therapeutic implications. Curr Genomics 8: 271-306, 2007.

18. McDowell KA, Riggins GJ and Gallia GL: Targeting the AKT pathway in glioblastoma. Curr Pharm Des 17: 2411-2420, 2011.

19. Vivanco I and Sawyers CL: The phosphatidylinositol 3-kinaseAKT pathway in human cancer. Nat Rev Cancer 2: 489-501, 2002.

20. Keunen O, Johansson M, Oudin A, Sanzey M, Rahim SA, Fack F, Thorsen F, Taxt T, Bartos M, Jirik R, et al: Anti-VEGF treatment reduces blood supply and increases tumor cell invasion in glioblastoma. Proc Natl Acad Sci USA 108: 3749-3754, 2011.

21. Phung TL, Ziv K, Dabydeen D, Eyiah-Mensah G, Riveros M, Perruzzi C, Sun J, Monahan-Earley RA, Shiojima I, Nagy JA, et al: Pathological angiogenesis is induced by sustained Akt signaling and inhibited by rapamycin. Cancer cell 10: 159-170, 2006.

22. Zhai X, Liang P, Li Y, Li L, Zhou Y, Wu X, Deng J and Jiang L: Astrocytes regulate angiogenesis through the Jagged1-mediated notch 1 pathway after status epilepticus. Mol Neurobiol 53: 5893-5901, 2016

23. Eccles SA: Parallels in invasion and angiogenesis provide pivotal points for therapeutic intervention. Int J Dev Biol 48: 583-598, 2004.

24. Dejana E, Tournier-Lasserve E and Weinstein BM: The control of vascular integrity by endothelial cell junctions: Molecular basis and pathological implications. Dev Cell 16: 209-221, 2009.

25. Wallez Y and Huber P: Endothelial adherens and tight junctions in vascular homeostasis, inflammation and angiogenesis. Biochim Biophys Acta 1778: 794-809, 2008.

26. Posada-Duque RA, Barreto GE and Cardona-Gomez GP: Protection after stroke: Cellular effectors of neurovascular unit integrity. Front Cell Neurosci 8: 231, 2014.

27. Dudley AC: Tumor endothelial cells. Cold Spring Harb Perspect Med 2: a006536, 2012.

28. Severson EA, Lee WY, Capaldo CT, Nusrat A and Parkos CA: Junctional adhesion molecule a interacts with afadin and PDZ-GEF2 to activate Rap1A, regulate beta1 integrin levels and enhance cell migration. Mol Biolo Cell 20: 1916-1925, 2009.

29. Fournier G, Cabaud O, Josselin E, Chaix A, Adélaïde J,Isnardon D, Restouin A, Castellano R, Dubreuil P, Chaffanet M, et al: Loss of AF6/afadin, a marker of poor outcome in breast cancer, induces cell migration, invasiveness and tumor growth. Oncogene 30: 3862-3874, 2011

30. Tawa H, Rikitake Y, Takahashi M, Amano H, Miyata M, Satomi-Kobayashi S, Kinugasa M, Nagamatsu Y, Majima T, Ogita $\mathrm{H}$, et al: Role of afadin in vascular endothelial growth factor-and sphingosine 1-phosphate-induced angiogenesis. Cir Res 106: 1731-1742, 2010.

31. Karar J and Maity A: PI3K/AKT/mTOR pathway in angiogenesis. Front Mol Neurosci 4: 51, 2011.

32. Prasad G, Sottero T, Yang X, Mueller S, James CD, Weiss WA, Polley MY, Ozawa T, Berger MS, Aftab DT, et al: Inhibition of $\mathrm{PI} 3 \mathrm{~K} / \mathrm{mTOR}$ pathways in glioblastoma and implications for combination therapy with temozolomide. Neuro Oncol 13: 384-392, 2011. 